\title{
Selected forms of therapy for individuals with autism spectrum disorder
}

\author{
Ewa Dudzinska ${ }^{1 \star}$, Kinga Szymona $^{2,3}$, Anna Pacian ${ }^{1}$, Teresa Kulik ${ }^{1}$
}

\author{
${ }^{1}$ Public Health Department, Nursing and Health Sciences Faculty, Medical University of Lublin, Poland \\ ${ }^{2}$ Children's University Hospital, Mental Health Outpatient Clinic, Lublin, Poland \\ ${ }^{3}$ Centre for Autism Therapy 'Alpha', Non-Public Health Care Clinic, Lublin, Poland
}

\begin{tabular}{l}
\hline ARTICLE INFO \\
\hline Received 02 February 2015 \\
Accepted 20 February 2015 \\
\hline $\begin{array}{l}\text { Keywords: } \\
\text { autism spectrum disorder, } \\
\text { therapy, } \\
\text { pharmacological treatment. }\end{array}$
\end{tabular}

\begin{abstract}
Autism spectrum disorder (ASD) is a condition of multiple origins. It is characterised by a range of behaviour patterns, in addition to disturbed social and emotional functioning. Of note, early therapy is conducive to better treatment results. A few recently discussed therapies have a particularly positive impact on children with ASD. Corbett et al. [2] proposed Sense Theatre. This involves instilling appropriate behaviours and communication patterns into the afflicted individual through acting. Role-playing and other similar techniques also offer an opportunity for children with ASD to improve their areas of empathy and social cooperation. With regard to bio-feedback-related techniques, Friedrich et al. [3] was noted for developing the Brain-computer method, a system of game interface connected to an external device. The method targets the mirror neuron system (MNS) in order to enhance cognitive, emotional and behavioural functions through neurofeedback. An approach put forward by Solomon et al. [10] is called 'Play and Language for Autistic Youngsters (PLAY) Project Home Consultation'. Herein, volunteers visit patients' homes on a regular basis to engage the children in play and games, after which they discuss with parents, the issues that came up. The PLAY reduces guardians' stress levels and improves children's skills. A pharmacological method is that of administering sulphoraphane [9], which reduces damaging effects. As others claim $[8,1,7]$, other dietary approaches prove efficient as well. In summation, an early intervention and the employment of a multimodal treatment approach can be of importance for enhancing the life of ASD-affected children.
\end{abstract}

\section{INTRODUCTION}

In the etiology of pervasive developmental disorders (following the DSM-5 classification - autism spectrum disorder, ASD), genetic and biologic factors play an important role. With regard to autism, environmental factors such as teratogenic and perinatal impairments may also prove significant. Additionally, it cannot be excluded that a particular child's possessed psychological factors which in coinciding with other genetic predispositions, may be of some importance in the etiology of autism [4].

The disorder is characterised by deficits in the areas of social and emotional functioning, as well as in the verbal and non-verbal communication strategies important in

\footnotetext{
* Corresponding author

e-mail: ewajoc@umlub.pl;

tel.: +48 814486720
}

social interactions. Moreover, within the afflicted, difficulties arise in developing and maintaining relations in various contexts. Furthermore, the presence of repetitive, stereotypical patterns of behaviour and interests is evident. Of note, individuals with autism often display impaired recognition and understanding of, and, hence, have difficulty reacting to, other people's emotional states (their thoughts, convictions and intentions) [6]. Research demonstrates that early intervention (timely therapy) results in the improvement of the state of individuals affected by autism [11]. According to the estimates of the Center for Disease Control and Prevention in the USA, an average of 1 out of 68 children has ASD [5]. This article offers a survey of selected treatment methods currently discussed in world literature which have a particularly positive impact on the functioning of children with ASD. 


\section{MATERIALS AND METHODS}

The utilised literature was obtained by web-searching in 2014, the articles found in the Medline resource website.

In the search strategy, the following keywords were employed: "ASD", "autism", "therapy" and "pharmacological treatments". We then selected and critically reviewed the most relevant 4 studies.

\section{RESULTS AND DISCUSSION}

A hallmark characteristic of individuals with ASD, is the impairment of mutual social interactions. This may have an impact on a variety of areas of functioning, including verbal and non-verbal communication, imitation and developing age-appropriate emotional relationships. In successfully dealing with ASD, it is, therefore, important to introduce therapeutic methods contributing to the accurate recognition of a range of emotions, and which include peer-group activities. One of the techniques described by Corbett et al. (2014) [2] which considerably influences the acquisition of significant social skills (such as peer and other interactions), is the new programme Sense Theatre. This engages healthy peers in the therapy.

Acting is an interactive process encompassing many aspects of socialising, namely observation, perception, interpretation and expressing thoughts and ideas. An actor has to, for example, pay attention to other participants, listen and respond to their signals, as well as to the thoughts and feelings they express. Acting, therefore, involves empathy and intentions, both of which are significant and problematic elements evidenced in ASD affected individuals. What is more, as an actor has to become familiar with the character he or she portrays, with the feelings and convictions displayed therein; the process leads to the broadened awareness of, and the improved familiarity with the experiences of other people. Thus, through acting, ASD-affected children can develop a deeper insight into, and a greater possibility of attributing the mental states of others onto themselves. Indeed, studies show that incorporating various theatrical methods considerably improve the relevant areas of functioning such as empathy and perspective-taking. The Sense Theatre programme applies varied acting techniques like role playing, scripts and improvisations which provide ASD-affected participants an opportunity to explore and practice skills of social cooperation in a safe and friendly environment. Together with their peers, they are involved in theatre-like games, thanks to which they are able to interact in a group. In the Sense Theatre method, young healthy peers are seen as 'exemplars' - serving as models for ASD-affected individuals to follow. The program is, thus, based on the notion that learning by way of exemplars raises possibilities of gaining skills, as modeling behavioural patterns on that of exemplars and interacting naturally with such individuals, is more effective and improves the likelihood of developing appropriate social skills.

Children with ASD require clear, repetitive and correct examples of behaviours in social relations. This can be provided by way of played roles and drama-based exercises. What is more, improvising and playing theatrical roles stimulates imagination and is conducive to a more flexible thinking and behaviour choice. Moreover, peers' positive reinforcement can enhance social motivation towards engaging in positive closer relations with other people. As individuals with ASD show deficits in social and communication skills (including imitation, empathy and shared attention), as well as restricted interests and repetitive patterns of behaviours, these deficits substantially impair satisfactory social interactions and prevent children from establishing normal relationships with family or friends.

With regard to the work of Friedrich et al. (2014) [3], their innovative therapy method, ,Brain-computer' is a game interface allowing for the brain and an external appliance to communicate. Brain-computer is, therefore, built upon the notions of neurofeedback (NFT) and biofeedback connections. Friedrich et al. point to the fact that the origins of autism are complex and the factors are numerous, and autism can be traced back to the presence within the individual of neurobiological disorders related to abnormally functioning areas of brain in the mirror neuron system (MNS) [3].

The research of Friedrich et al. [3] suggests that both hyperactivity of neuronal connections and hypoactivity of neuronal connections in children with ASD, particularly in the MNS region, correlates with the efficiency of cognitive, emotional and behavioral functions. Friedrich et al. note that applying neurofeedback techniques induces neuroplastic changes and leads to the normalisation of MNS regions. Indeed, children with attention deficit hyperactivity disorder, but also ASD, show improvement in the behavioural area due to the neuro-regulation of relevant brain structures.

Solomon et al. (2014) [10] describe a programme dedicated to young children with ASD called 'Play and Language for Autistic Youngsters (PLAY) Project Home Consultation'. It is intended for children aged 2-8 year old. In the project, trained consultants seek to support the parent-child relationship by means of an organised approach involving coaching, modeling, applied methodology and learning games, supplemented with the use of video material. PLAY consultants visit each family once a month for 3 hours, of which, 15 minutes is devoted to video games and play; then the influence of the undertaken play and applied effective methodological approaches is analysed with the parents. During the visits, the trained consultants provided parents with help in identifying subtle and problematic autistic symptoms, as well as ways of initiating appropriate treatment and remedial action in order to achieve improvement. The play activity is tailored both to the individual child and to the child's stage of development.

The analysis includes the 'PLAY Plan', which delimits the approaches, defining techniques and activities contributing to providing parental opportunities and skills in effectively supporting their child's functional development. Of note, generally, 12 months after launching the programme, a substantial stress levels reduction in the guardians of ASDaffected children, as well as the alleviation of depression symptoms are usually observed.

A number of papers reveal that dietary treatment [8], supplementation by omega-3 acids [1] and exclusion of some nutrients (gluten-free and milk-free diet) [7] have an effect on ASD prognosis. Among such papers, the highly 
interesting research carried out by Singh et al. (2014) [9] relates to administering sulphoraphane (isothiocyanate), a substance derived from broccoli and other cruciferous vegetables.

Singh et al. note that sulphoraphane counteracts many biochemical and molecular disturbances related to autism, among these being oxidative stress and restrained antioxidant capacity, glutathione synthesis defects, mitochondrial dysfunctions and low oxidative phosphorylation, increased lipid peroxidation and neuro-inflammation. Although it is not clear whether the abnormalities result from the etiology of the disorder or rather these are secondary symptoms, Singh et al. demonstrate that correcting the abnormalities often improves behaviour in ASD cases.

The work of Singh et al. reveal that sulphoraphane can ameliorate many unrelated genetic disorders by activating the 'stress proteome' which regulates a number of aforementioned damaging processes. Moreover, sulphoraphane, as well as hydroxyurea, phenylbutyrate and trichostatin A show theurapeutic potential towards re-establishing regular cellular homeostasis in many unrelated genetic disorders. As the researchers highlight, sulphoraphane is a phytochemical. Isothiocyanates such as sulphoraphane occur in the plant in the form of precursors, the so-called glucosinolates. Sulphoraphane is released when its precursor, glucoraphanin undergoes the hydrolysis induced by myrosinase - an enzyme co-occurring with glucosinolates in cruciferous vegetables.

Depending on the purpose, sulphoraphane can be approved for use as a diet supplement or medication. Thus, it is rightly believed to be a well-tolerated substance of low toxicity. Furthermore, sulphoraphane was shown to produce an effect similar to that of an acquired fever within the organism. Numerous studies indicate that fever can temporarily alleviate the disturbed behaviour in many autistic patients. It is worth noting that the level of enhancement (usually of stereotypical behaviours and communication disorders) was related to the presence of a higher fever among subjects with autism.

In the conducted research, sulphoraphane was administered to men (n-29) diagnosed with moderate and serious ASD (doses were relevant to the subject's weight and amounted to 9-27 mg). After 4, 10 and 18 weeks from start of treatment, and 4 weeks after completing therapy, the results, when compared to the placebo group, demonstrated an improvement of behavioural symptoms. The enhancement lasted until the treatment was discontinued.

The study results have been compared by way of the use of the Clinical Global Impression scale. This revealed that $46 \%$ of the participants who have been receiving medication, noticeably improved their social interaction skills, while $44 \%$ ameliorated their abnormal behaviours such as irritability, repetitive movements and hyperactivity, and $42 \%$ made progress in verbal communication. Hence, it can be said that the group receiving sulphoraphane exhibited improvement in social interactions, and in emending disturbed behaviour and miscommunication. Unfortunately, the positive changes disappeared after the treatment was discontinued.

The multifactor etiology of ASD should be the point of departure for many methods and forms of therapies followed in therapeutic programmes. It is for this reason that subsequent research is needed to better understand the etiology of autism spectrum disorder and to elaborate more efficient intervention methods for the best therapy results [11]. It is noteworthy that an early diagnosis and the introduction of a multimodal therapy (speech therapist, psychologist, teacher) and dietician) which would involve innovative approaches, can substiantially improve the prognosis of individuals with autism spectrum disorder.

\section{CONCLUSIONS}

1. In the light of current research, there is no one versatile treatment for ASD-affected children.

2. Due to the higher incidence of ASD in recent years, it is important to investigate its origins, as well as to seek and implement new innovative methods of therapy for individuals with autism spectrum disorder.

3. Despite lacking evidence of their alleviating effects on autism symptoms, the therapeutic and pharmacological treatments necessitate further research to ascertain their efficiency.

\section{REFERENCES}

1. Bent S., Bertoglio K., Hendren R.L.: Omega-3 Fatty Acids for Autistic Spectrum Disorder: A Systematic Review. J Autism Dev Disord., 39(8), 1145, 2009

2. Corbett B.A., Qualls L.R. Valencia B., Fecteau S.M., Swain D.M.: Peer-mediated theatrical engagement for improving reciprocal social interaction in autism spectrum disorder. Front Pediatr., 10, 110, 2014

3. Friedrich E.V.C., Suttie N., Sivanathan A., Lim T., Louchart S., Pineda J.A.: Brain-computer interface game applications for combined neurofeedback and biofeedback treatment for children on the autism spectrum. Front Neuroeng., 3, 7, 21, 2014

4. Komender J., Jagielska G., Bryńska A., editors (2012). Autyzm i zespół Aspergera. Warszawa: Wydawnictwo Lekarskie PZWL; p. 17.

5. Ling-Yi Y.: Quality of life of Taiwanese adults with autism spectrum disorder. PLoS One, 9,9,10, 2014.

6. Mazza M., Pino M.C., Mariano M., Tempesta D., FerraraM., De Berardis D, Masedu F., Valenti M.: Affective and cognitive empathy in adolescents with autism spectrum disorder. Front Hum. Neurosci., 8, 2014.

7. Millward C., Ferriter M., Calver S. J., Connell-Jones G.G.: Glutenand casein-free diets for autistic spectrum disorder. Cochrane Database Syst. Rev., 2008; (2): CD003498.

8. Napoli E., Dueñas N., Giulivi C.: Potential Therapeutic Use of the Ketogenic Diet in Autism Spectrum Disorders. Front Pediatr.,2, 2014.

9. Singh K., Connors S.L., Macklin E.A., Smith K.D., Fahey J.W., Talalay P., Zimmermana A.W.: Sulforaphane treatment of autism spectrum disorder (ASD). Proc. Natl. Acad. Sci. USA, 111, 43, 15550, 2014.

10. Solomon R.,Van Egeren L.A., Mahoney G., Quon Huber M. S., Zimmerman P.: PLAY Project Home Consultation Intervention Program for Young Children With Autism Spectrum Disorders: A Randomized Controlled Trial. J Dev BehavPediatr., 35(8), 475, 2014

11. Weitlauf A.S., Mc Pheeters M.L., Peters B., Sathe N., Travis R.,Aiello R., Williamson E., Veenstra-VanderWeele J., Krishnaswami S., Jerome R., Warren Z.: Therapies for children with autism spectrum disorder. Behavioral Interventions Update. Agency for Healthcare Research and Quality (US), Comparative Effectiveness Review, 137, 2014. 